\section{Antivirals pay the Toll}

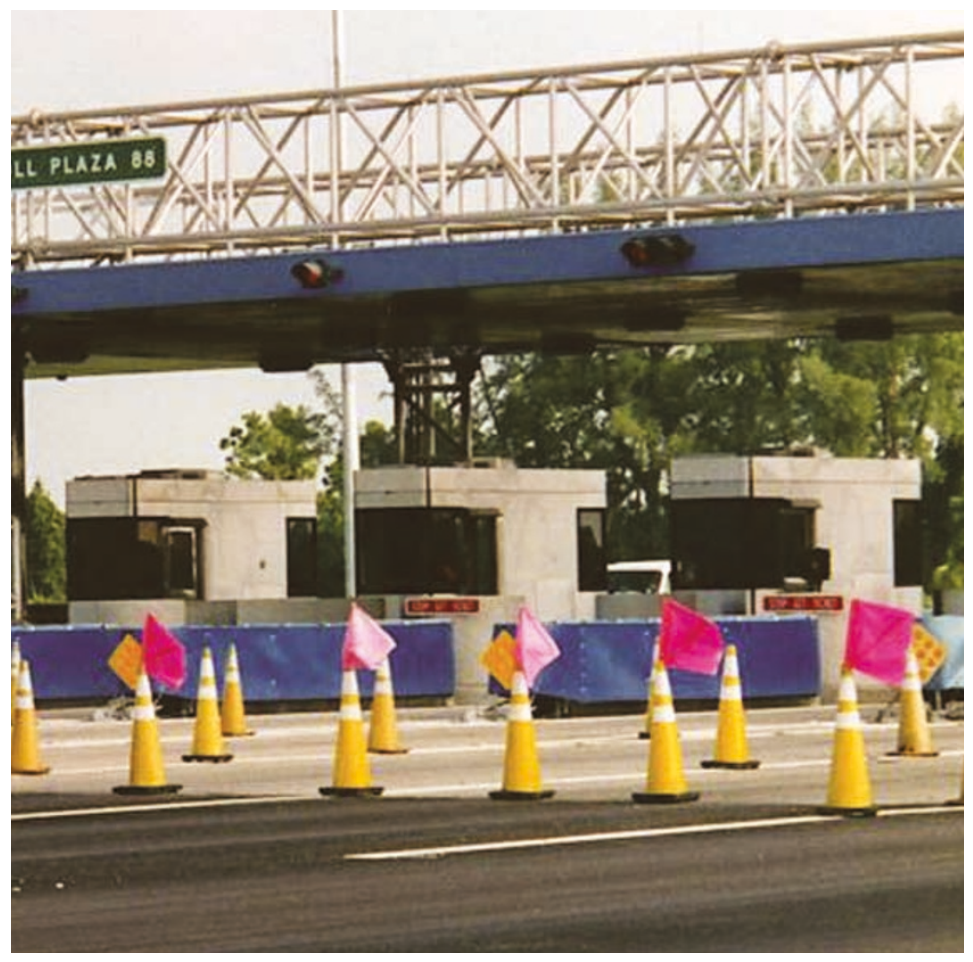

Most of the antiviral agents that are either available, such as acyclovir and famciclovir, or in development, inhibit pathways that are involved in viral replication or assembly. By contrast, the antiviral drug imiquimod, which is an imidazoquinoline amine, is an immune-response modifier that activates immune function. In the February issue of Nature Immunology, Shizuo Akira and colleagues show that imidazoquinolines activate immune cells by means of the Toll-like receptor 7 (TLR7) signalling pathway, which leads to the induction of interferon- $\alpha$ and other inflammatory cytokines.

Toll-like receptors (TLRs) are named from the Toll gene of Drosophila melanogaster. As well as being involved in development, TLRs are also a vital component in the fly's immune response to infections. So far, ten mammalian TLRs have been reported, each essential for recognition of specific bacterial, viral and fungal components. They associate with a protein, MYD88, which acts as an adaptor that leads to activation of both the transcription factor NF- $\mathrm{KB}$ and the JNK MAP kinase. So, TLRs link microbial products to transcription, and the immune response is set in motion. Akira and colleagues showed in vivo that imiquimod and resiquimod, a related compound, activate immune cells in a TLR7- and MYD88-dependent manner. Furthermore, neither Tlr7- nor Myd88-deficient mice showed inflammatory cytokine production by any immune cells, or maturation of immune-initiator dendritic cells in response to imidazoquinolines. Mature dendritic cells are crucial for inducing immune responses by displaying small amounts of foreign components to effector T cells.

The more potent resiquimod is undergoing Phase III clinical trials at present for genital herpes. The continuing trials indicate that resiquimod increases the time between genital herpes outbreaks, and could produce a long-lasting suppressive effect without the need for daily therapy. Through its novel approach of activating the immune system as if it is a vaccine, some clinical investigators believe resiquimod might reduce viral transmission and, with several courses of therapy, place the disease into clinical remission.

\section{Cycle stopper}

Cyclin-dependent kinases (CDKs) have key roles in the regulation of the cell cycle. Aberrant activity of CDKs is frequently implicated in cancer, so CDK inhibition could be a promising antitumour strategy; indeed, several non-specific CDK inhibitors are already in clinical trials. However, targeting a single CDK to cause cell-cycle arrest in a particular phase could be more desirable; for example, suppression of tumour growth by arrest in the G1 phase of the cycle could reduce the stress for normal cells, as they spend most of their time resting in the G0-G1 phase. But selectively inhibiting kinases is a considerable challenge owing to the high homologies of their active sites. Addressing this issue by using structurebased design, Honma et al. now present two papers on the development of a new class of selective CDK4 inhibitors that cause cell-cycle arrest in the G1 phase.
Taking the crystal structure of activated CDK2 as a basis for a model of CDK4, a new de novo design strategy was used to identify promising drug scaffolds. Commonly, the structures that are obtained from de novo design progams are difficult to synthesize and their components are not readily available. So, to counteract any such potential problems, the authors constructed a program to extract essential parts of the structures and check their availability and/or synthetic accessibility. Scaffold candidates were then screened in a CDK4 inhibition assay, and one hit was chosen as the basis for library synthesis. Structure-activity relationship studies and further molecular modelling allowed the binding mode of this series of lead compounds to be deduced, and a compound that potently inhibited CDK4 was identified.

But what about the selectivity? Although the identified compound was moderately ( $>50$-fold) selective over kinases not in the CDK family, it had comparable activity against $\mathrm{CDK} 1$ and CDK2. To address this problem, the authors used molecular modelling to identify the amino acids that were most specific for the active site of
CDK4, and then used de novo design based on the deduced binding mode to generate a library of compounds tailored to interact with these residues. As a result, a compound that was both a potent inhibitor of CDK4 and selective over other CDKs ( $>190$-fold) and other kinases ( $>530$-fold) was identified, and was found to cause cell-cycle arrest of a cancer cell line in the G1 phase.

Such structure-based approaches should accelerate the development of CDK4 inhibitors as a potential new class of cytostatic antitumour agents. Furthermore, the integration of structure selection for synthetic accessibility with a de novo design strategy could find application in other rational drug design programs.

Peter Kirkpatrick

(2) References and links ORIGINAL RESEARCH PAPERS Honma, T. et al. Structure-based generation of a new class of potent Cdk4 inhibitors: new de novo design strategy and library design. inhibitors: new de novo design strategy and library design. A novel approach for the development of selective Cdk4 inhibitors: library design based on locations of Cdk4 specific amino acid residues. J. Med. Chem. 44, 4628-4640 (2001) FURTHER READING Ikuta, M. et al. Crystallographic approach to identification of cyclin-dependent kinase 4
(CDK4)-specific inhibitors by using CDK4 mimic CDK2 protein. J. Biol. Chem. 276, 27548-27554 (2001) 
Interferon- $\alpha$, which is produced by dendritic cells, is the body's natural defence against many viruses. In addition, certain subsets of dendritic cells express TLR7 and TLR9 exclusively out of the ten known TLR family members. The essential role of TLR7 in the response to these viral agents provides compelling evidence for its general involvement in antiviral host defence. This mechanistic understanding that imidazoquinolines stimulate the immune system through TLR7 is welcome news for the future design of antivirals based on TLR7 ligands. This work raises new questions about whether TLR7 interacts directly with viruses on infection. Furthermore, because each Toll receptor recognizes different microbial components, these receptors might be useful as specific antibacterial and antifungal targets.

Melanie Brazil

(D) References and links ORIGINAL RESEARCH PAPER Hemmi, $\mathrm{H}$. et al. Antiviral low molecular weight compounds activate immune cells via TLR7-MyD88dependent signalling pathway. Nature Immunol. 2002 Jan 22 (DOI 10.1038/ni758) FURTHER READING Akira, S. et al. Toll-like receptors: critical proteins linking innate and acquired immunity. Nature Immunol. 2, 675-680 (2001)

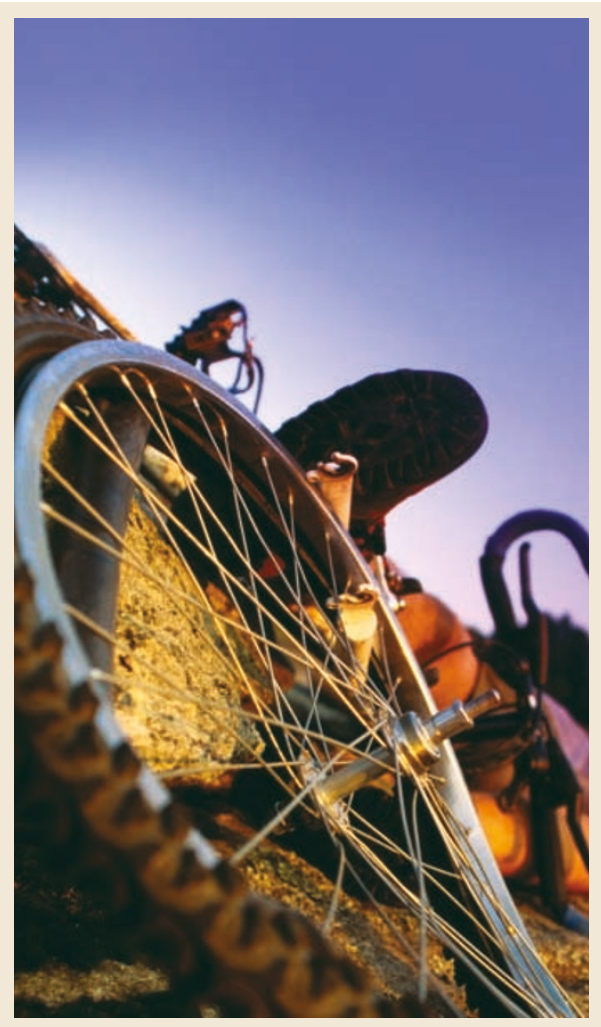

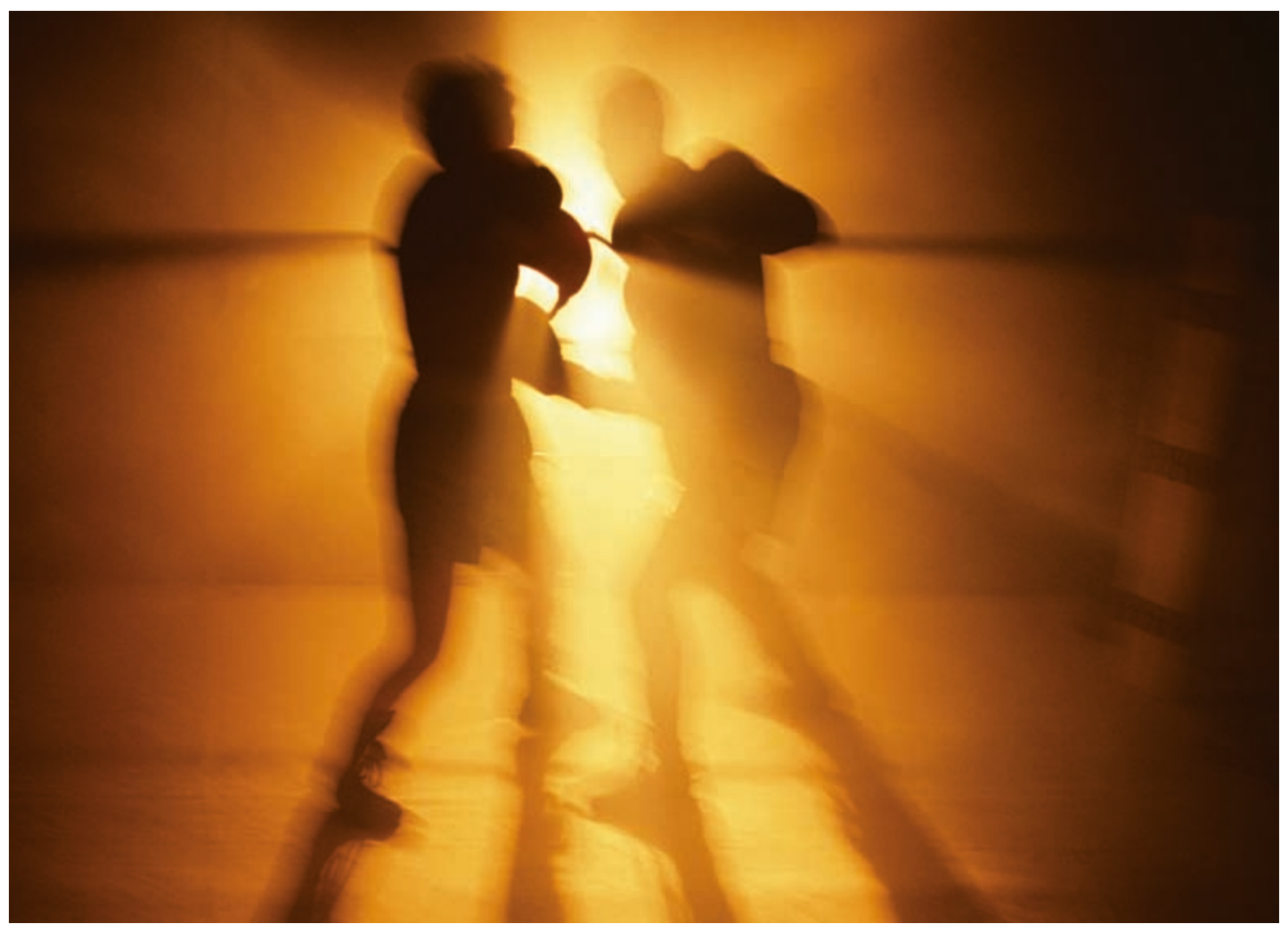

\section{DRUG RESISTANCE}

\section{Hit where it hurts}

Protease inhibitors are a key part of many treatment regimes for AIDS, but rapid evolution of resistance can limit their effectiveness. Using crystal structures of human immunodeficiency virus (HIV) protease and its complexes with inhibitors, in combination with clinical resistance data, Wei Wang and Peter Kollman now suggest a strategy to combat this problem. Analysis of the energetics of binding and variability at each sequence position of HIV protease indicates that designing drugs to interact more strongly with highly conserved residues could limit the development of resistance.

The authors assessed the variability of HIV protease residues using multiple alignments of the sequences of 80 related viral proteases from different species. Low variability indicates that a residue is well conserved, and so might be catalytically or structurally important. Indeed, comparison with known resistance mutants revealed that no single drug-resistance mutations have been observed at residues with variability below a certain threshold; these residues include those known to be crucial for catalysing peptide cleavage.

But what is the molecular basis of resistance? To identify residues crucial for ligand binding, structural data for HIV protease bound to a substrate analogue and to each of the five FDAapproved protease inhibitors were used in molecular-modelling calculations of the binding energetics for each residue. Next, the difference between the contribution of each residue to drug binding and to substrate binding was evaluated. Taking these data together with the residue variability statistics revealed that single drugresistance mutations often occur at poorly conserved residues that interact more favourably with the drug than with the natural substrate. Wang and Kollman thus suggest the following mechanism for drug resistance: if drug binding depends on an interaction with a poorly conserved residue that is more favourable than the corresponding interaction of the normal substrate with the residue, then mutation of the residue can lead to resistance by significantly reducing drug binding without affecting protease function. So, resistance-evading drugs should be designed to interact strongly with highly conserved residues. On the basis of the interactions of the current protease inhibitors with their target, the authors suggest several alterations that might allow resistance to be overcome, which could be a starting point for a medicinal chemistry programme.

Furthermore, the authors propose an empirical parameter that is defined as the product of a residue's contribution to the binding energy and the variability of that residue, which can be used for the quantitative prediction of mutations that cause drug resistance, as indicated by its $76 \%$ success rate with the drugs analysed. The parameter and the approach that the authors use for decomposing binding contributions in terms of individual residues are general, and so could potentially be used to assist the design of resistance-evading drugs for any target.

Peter Kirkpatrick

(2) References and links ORIGINAL RESEARCH PAPER Wang, W. \& Kollman, P. A. Computational study of protein specificity: the molecular basis of HIV-1 protease drug resistance. Proc. Natl Acad. Sci. USA 98, 14937-14942 (2001)

FURTHER READING Wang, W. et al. An analysis of the interactions between the Sem-5 SH3 domain and its ligands using molecular dynamics, free energy calculations, and sequence analysis.

J. Am. Chem. Soc. 123, 3986-3994 (2001) | De Clercq, E. Strategies in the design of antiviral drugs. Nature Rev. Drug. Discov. 1, 13-25 (2001) 